\title{
Design and build a compact Raman sensor for identification of chemical composition
}

\author{
Christopher S. Garcia ${ }^{a}$, M. Nurul Abedin ${ }^{b}$, Syed Ismail ${ }^{b}$, Shiv K. Sharma ${ }^{c}$, Anupam K. Misra ${ }^{c}$, \\ Stephen P. Sandford ${ }^{b}$, and Hani Elsayed-Ali ${ }^{\mathrm{a}}$ \\ aOld Dominion University, Hampton Blvd, Norfolk, VA, 23529 \\ ${ }^{b}$ NASA Langley Research Center, Hampton, VA, 23681 \\ ${ }^{c}$ Hawaii Institute of Geophysics and Planetology, University of Hawaii \\ 1680 East-West Rd., POST 602, Honolulu, HI 96822
}

\begin{abstract}
A compact remote Raman sensor system was developed at NASA Langley Research Center. This sensor is an improvement over the previously reported system, which consisted of a $532 \mathrm{~nm}$ pulsed laser, a 4-inch telescope, a spectrograph, and an intensified CCD camera. One of the attractive features of the previous system was its portability, thereby making it suitable for applications such as planetary surface explorations, homeland security and defense applications where a compact portable instrument is important. The new system was made more compact by replacing bulky components with smaller and lighter components. The new compact system uses a smaller spectrograph measuring $9 \times 4 \times 4$ in. and a smaller intensified CCD camera measuring 5 in. long and 2 in. in diameter. The previous system was used to obtain the Raman spectra of several materials that are important to defense and security applications. Furthermore, the new compact Raman sensor system is used to obtain the Raman spectra of a diverse set of materials to demonstrate the sensor system's potential use in the identification of unknown materials.
\end{abstract}

Keywords: Raman spectroscopy, pulsed laser, ICCD, remote Raman.

\section{INTRODUCTION}

Raman spectroscopy was established to be an ideal tool for determining chemical composition and structure of a given material due to the following reasons: 1) it is a nondestructive method with no sample preparation required, which allows analysis of rare and valuable samples; 2) it can detect both organic and inorganic materials in solid, liquid or gas phase; 3) Raman spectra of molecules consist of sharp, well-defined lines that can be used as fingerprints for the unambiguous identification of polyatomic ions and molecules; and 4) various polymorphs can be specified, and crystalline and mineral glasses formed from melt or by impact can be differentiated due to the uniqueness of the Raman spectral features of various polymorphs of the same mineral. ${ }^{1-2}$

For many applications such as planetary surface analysis and homeland security, a portable Raman system is essential, because analyses of samples usually need to be performed on the spot. ${ }^{3-7}$ Both in situ Raman spectroscopy and remote Raman spectroscopy have been extensively studied for a wide range of applications. However, a remote system was chosen for its several additional benefits to planetary surface analysis and homeland security applications, as compared to an in situ system. When integrated into a Martian or lunar lander or rover, a remote Raman system has a greater operational radius, provides access to a larger available rock sample population, allows a quick selection of samples by scanning the area for targets of interest, and reduces travel time and power consumption of the rover or lander. In addition for homeland security scenarios, a remote Raman system will allow analysis of samples that are hazardous or highly combustible.

A compact remote Raman sensor system was developed at NASA Langley Research Center Raman Spectroscopy Lab and has been previously reported. ${ }^{6,7}$ The Raman system is capable of obtaining Raman spectra of various rocks and 
minerals from a distance of the order of tens of meters from the sample. Furthermore, the system is also capable of operating in daylight or in environments with high background radiation by utilizing a pulsed laser and a gated detector.

One of the primary design objectives in developing this Raman system is to minimize the size and weight of the entire instrument by utilizing smaller instruments and a minimal number of components. This paper describes improvements made to the spectrograph and detector sections of the Raman sensor system, and compares the performance of the improved compact system to that of the previous system.

\section{EXPERIMENTAL SETUP AND PROCEDURE}

The improved compact remote Raman sensor system is depicted in the schematic diagram in Figure 1. Detailed description of the previous system, before a smaller spectrograph and smaller intensified charge-coupled devices (ICCD) camera were added, can be found elsewhere. ${ }^{6,7}$

The excitation source is a mini Nd:YAG pulsed laser from Big Sky Laser (model UltraCFR) that produces a $532 \mathrm{~nm}, 23$ $\mathrm{mJ} /$ pulse at pulse repetition frequencies up to $20 \mathrm{~Hz}$ frequency, and 8ns pulse width. Coaxial configuration is achieved by making the laser beam collinear to the telescope axis using two $45^{\circ}$ prisms. This configuration results in maximum performance by allowing collection of the $180^{\circ}$ backscattered signal from the target to the telescope. The sample is located $5.6 \mathrm{~m}$ from the system. A telescope collects the backscattered signal from the target. The telescope is a Maksutov-Cassegrain telescope with 4-inch aperture (MEAD ETX-105).

In the previous system, the telescope output is focused into the entrance aperture of the spectrograph (Kaiser Optical Systems, Inc. (KOSI), Holospec f/1.8i). Inside this spectrograph, the signal is passed through a notch filter (KOSI 532 $\mathrm{nm}$ Holographic SuperNotch-Plus filter, 2-inch diameter) to attenuate the strong Rayleigh signal, and then focused onto a $50 \mu \mathrm{m}$ slit which defines the resolution of the spectral lines. The resulting signal is then collimated and passed through a holographic transmission grating (Kaiser Optical Volume Phase Holographic transmission grating) to produce the spectrum of the signal. With the holographic transmission grating, the spectral range of the spectrograph is doubled without sacrificing resolution. This result is possible because the grating simultaneously disperses two spectral tracks on the detector: the low frequency region of a spectrum is displayed on the upper half of the detector, whereas the high frequency portion is dispersed on the lower half. This spectrograph measures approximately $17 \times 6 \times 7 \mathrm{in}$.

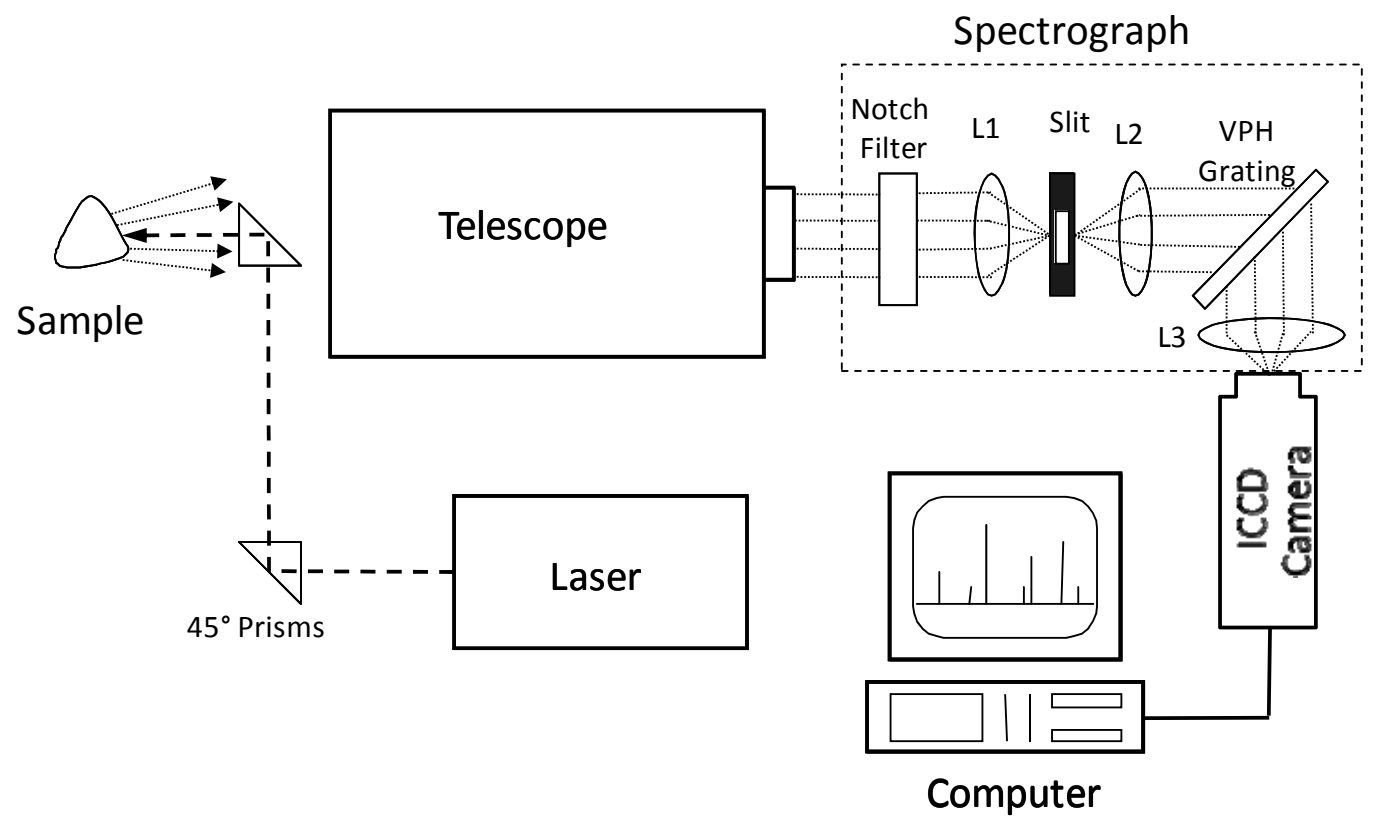

Figure 1. Schematic diagram of the improved compact Raman remote sensor system showing the smaller spectrograph and ICCD camera. 
For the compact system, the entire spectrograph is removed and replaced with smaller components as shown in Figure 1. To attenuate the strong Rayleigh-scattered signal portion of the telescope output, a 1 in. diameter KOSI $532 \mathrm{~nm}$ Holographic SuperNotch-Plus filter is placed along the path of the telescope output. The filtered signal is then focused using L1 onto a $100 \mu \mathrm{m}$ slit and then collimated by L2 onto the grating. The grating is a mini volume phase holographic transmission grating, which measures $1 \times 1.4$ in. Similar to the grating used in the previous spectrograph but only about a quarter in size, the grating also disperses two spectral tracks simultaneously onto the detector. This entire spectrograph section is contained in an enclosure measuring about $9 \times 4 \times 4$ in. This volume represents a mere $1 / 5$ of the volume of the previous spectrograph.

The spectrum produced by the spectrograph is imaged on an ICCD camera, which is directly coupled to the spectrograph. In the previous system, the camera used was a thermoelectrically-cooled and gated camera (Princeton Instruments PI-Max ICCD camera) with $1024 \times 256$ pixels and $26 \times 26 \mu \mathrm{m}$ pixel size. This camera provides superior gain of up to 250X making it possible to detect extremely weak Stokes lines. Furthermore, the gate width can be reduced to only a few nanoseconds, enabling an effective gated operation of the camera. The new camera measures about $4.5 \times$ $4.5 \times 12 \mathrm{in}$. and an electronic controller that provides power, timing signal, and signal processing to the camera measures about $6 \times 9 \times 14$ in.

For the improved compact system, the camera used is a mini ICCD camera (Photonic Science MiniFDI ICCD camera) with $1392 \times 1040$ pixels and $6.45 \times 6.45 \mu \mathrm{m}$ pixel size. This camera, which has an intensifier gain of up to $100 \mathrm{X}$, includes a high performance $18 \mathrm{~mm}$ diameter image intensifier to amplify the optical signal prior to its capture in the CCD. It features digitization of the CCD signal to 12 bits. A 16 bit digitization can be achieved by switching to Fusion mode which increases the saturation limit to 65,000 ADU (analog to digital unit). The only drawback of using the Fusion mode is that it takes longer time to acquire an image. The camera is controlled via an application software (MediaCybernetics Image-Pro Express Version 6.0) which is installed in the computer. This software allows control of such parameters as exposure time, intensifier gain level, number of accumulations, and triggering.

One of the important features of the previous Raman system is its ability to obtain Raman spectra during daytime or under room lights. ${ }^{6-8}$ This capability is absolutely necessary for planetary surface measurements, where the ambient light cannot be controlled but is needed in locating interesting minerals and geologic features. Moreover, when analyzing chemical spills, fume clouds, or toxic explosions, the measurements will be limited by the background if the Raman system is designed to only work in dark conditions. The ability to obtain Raman spectra under daylight was achieved by gating the camera so that it is only turned on right before the $8 \mathrm{~ns}$ laser pulse is fired. The gate, then turned off immediately after a laser pulse, is required to minimize the background radiation that would have been picked up by the camera when the laser pulse, and thus Raman signal, is not present. In addition, the PI-Max camera was also capable of accumulating any number of shots by software (instead of accumulating signal on the CCD, which saturates at 65,000 Analog to Digital Units (ADU). Use of gating and the accumulation capability of the camera allows for measurements with longer exposure time interval. This longer interval results in high quality spectra with minimized background and without saturating the CCD. For example, to take measurements with 1 second exposure time, the camera is set for 20 accumulations (corresponding to 20 pulses per second frequency of the laser), and a gate width of 22 ns. During the 1 second exposure, the camera is only turned on for 22 ns multiplied by 20 accumulations, or a total of only $440 \mathrm{~ns}$. Therefore, the total time that the CCD can pick up background radiation is only a very minute fraction of the exposure time. For materials that produce lower Raman signal, the quality of the spectra can be improved by increasing the number of accumulations accordingly.

The mini ICCD camera in the improved compact system, as depicted in Figure 1, also allows gated operation and accumulation of signal with some limitations. For gating, the shortest integration time allowed by the application software is $1.0 \mathrm{~ms}$. Because there is no other timing generator used to gate the camera, this is also the shortest gate width that can be achieved. Background suppression is therefore not as effective as with the previous camera. For signal accumulation, the mini ICCD camera accumulates the signal on the CCD (not by software) and has a limit of only up to 16 accumulations. The total accumulated signal still saturates at 4096 ADU (or 65,000 ADU in Fusion mode), limiting the quality of spectra that can be achieved with the system, and making it necessary to turn off the room lights during experiment. 
The spectra produced by the mini ICCD application software is processed and analyzed using GRAMS/AI (Galactic Industries) software. Calibration of the horizontal axes of the spectra from pixel position to nanometers and wavenumber or $\mathrm{cm}^{-1}$ was done by obtaining the spectral lines of a Neon lamp with the system. The pixel positions of the measured Neon spectral lines were compared to corresponding standard values in nanometers.

\section{RESULTS AND DISCUSSION}

The performance of the improved compact remote Raman sensor system that utilizes a smaller spectrograph and mini ICCD camera was evaluated by comparing the spectra with those obtained with the previously reported system that used larger spectrograph and camera system.

Figure 2 shows the Raman spectra of isopropanol, acetone, naphthalene, and calcite obtained with the current compact system. Figure 3 shows the Raman spectra of some of the same materials obtained with the previous system. ${ }^{6,7}$ As seen in Figures 2 and 3, the Raman fingerprints of all the samples are present on the spectra obtained with the compact system as they are on the ones obtained with the previous system. The obvious difference in the two sets of spectra is that the ones obtained with the current system contain more significant amount of background signal. Unfortunately, background signal still appears on the spectra in Figure 2 even when the measurements were performed with the room lights turned off. This significant amount of background signal is mainly due to the long-lived fluorescence and other light sources in the room such as the computer screen, and it is still picked up by the camera because the integration time is too long compared to the width of the laser pulse.

In the calcite spectrum in Figure 2a, the measured spectral lines very closely correspond to the standard spectral lines of calcite. The standard NIST values of the Raman fingerprints of calcite are 155.5, 281.7, 711 and $1085 \mathrm{~cm}^{-1}{ }^{9}$ The very prominent line at $1085 \mathrm{~cm}^{-1}$ is due to the symmetric stretching mode of carbonate. For the other samples, the observed Raman lines are mostly within $\pm 2 \mathrm{~cm}^{-1}$ of the standard values. Some of the observed lines, however, deviate by up to 5 $\mathrm{cm}^{-1}$ from their standard values. These large errors can be attributed to the errors that can be introduced by the small size of the CCD pixels. Because the size of each pixel is only $6.45 \mu \mathrm{m}$ whereas the width of the slit used is $50 \mu \mathrm{m}$, the minimum possible width of a spectral line is at least 8 pixels. Also, each error of 1 pixel in the horizontal position of the observed data corresponds to about $1.5 \mathrm{~cm}^{-1}$ error in the calibrated data. Raman spectra can therefore be improved by using a narrower slit, but doing so will also block more Raman signal and degrade the spectra.

For naphthalene, the standard Raman bands are found at 510, 763.8, 1066, 1430, 1710.5, and $3056.4 \mathrm{~cm}^{-1}{ }^{9}$ Comparing these to the Raman bands from the two sets of observed data for naphthalene, the average errors are found to be only 1.81 and $2.78 \mathrm{~cm}^{-1}$ for the compact system and the previous system, respectively. For acetone, the standard Raman bands are found at 787, 1066, 1430, 1710.52922 , and $3005.5 \mathrm{~cm}^{-1}$, and comparing these values to the observed data results in average errors of only 2.31 and $2.5 \mathrm{~cm}^{-1}$ for the compact system and the previous system, respectively. ${ }^{9}$ The accuracy of the location of the Raman bands observed using the compact system is therefore comparable to that achieved with the previous system.

It should be noted that in Figure 2, despite the small error in the Raman shift position of the Raman bands, the Raman spectrum of each sample shows the fingerprint lines well enough to positively identify the materials if they were unknown.

Figure 4 depicts the high frequency region Raman spectra of water, ice and other hydrous minerals obtained with (a) the improved compact remote Raman sensor system, and (b) the previous Raman sensor system. The previous Raman sensor system has been demonstrated to have an excellent ability to measure the symmetric and antisymmetric stretching $\mathrm{O}-\mathrm{H}$ vibrational modes of water molecules that can be found in the $3100-3600 \mathrm{~cm}^{-1}$ region of their Raman spectra as can be seen in Figure $4 \mathrm{~b}$ in the spectra of water and ice. ${ }^{6,10}$ This ability is true even for other hydrous minerals, $\mathrm{FeSO}_{4} \cdot 7 \mathrm{H}_{2} \mathrm{O}$, $\mathrm{MgSO}_{4} \cdot 7 \mathrm{H}_{2} 0$, and $\mathrm{MgCl}_{2} \cdot 6 \mathrm{H}_{2} \mathrm{O}$, which show no physical evidence of containing any water. The observed Raman bands in the same region are due to stretching modes of water as previously stated, and indicate the presence of chemically bonded water molecules in these samples. The Raman sensor system is therefore an excellent instrument for detecting water, even in dry samples and environments which would otherwise show no sign of presence of liquid water. 

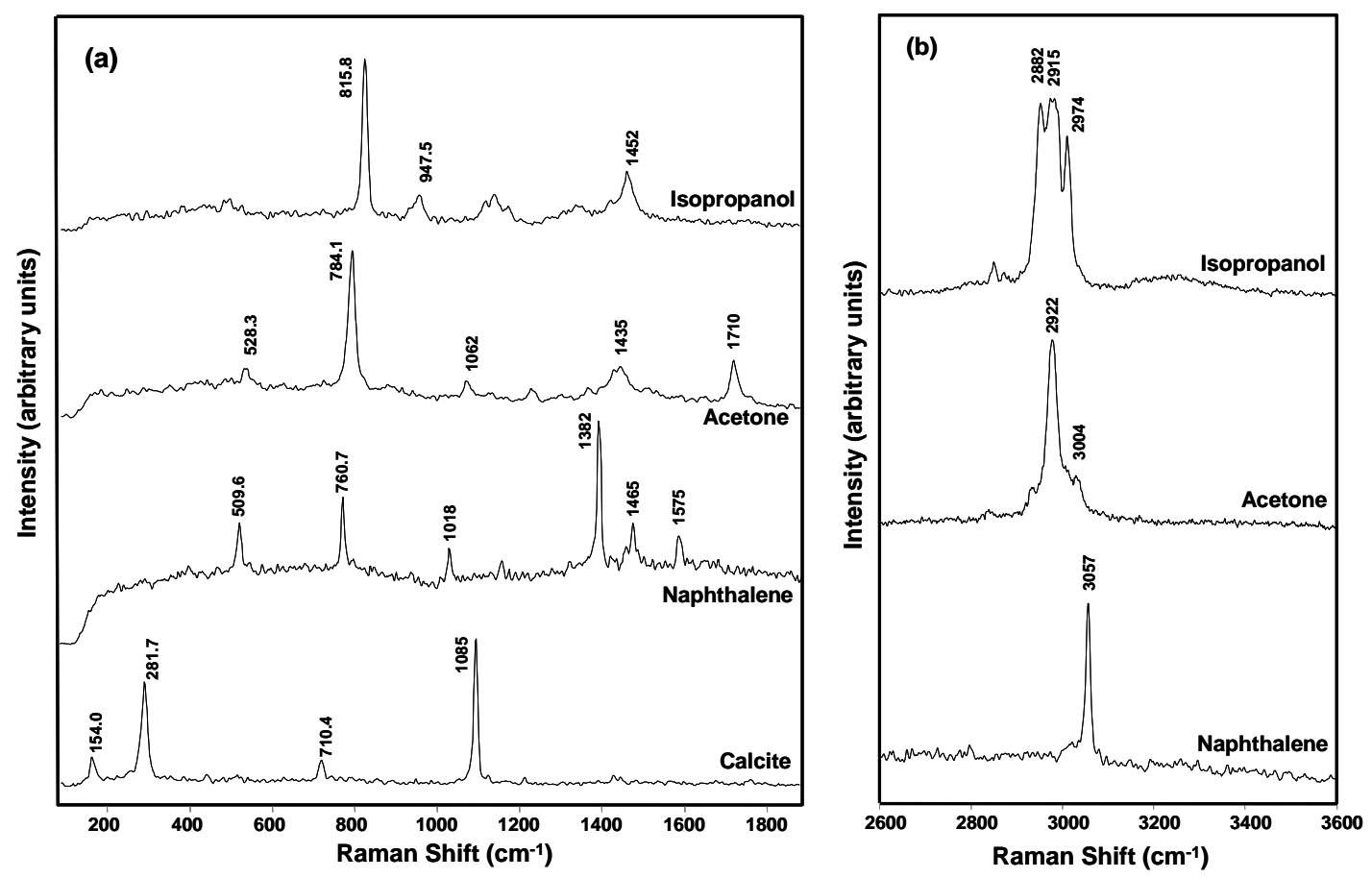

Figure 2. Raman spectra of isopropanol, acetone, naphthalene, and calcite in the (a) low frequency $\left(0-1900 \mathrm{~cm}^{-1}\right)$ and in the (b) high frequency $\left(2600-3600 \mathrm{~cm}^{-1}\right)$ frequency shift regions (obtained using the improved compact remote Raman sensor system).
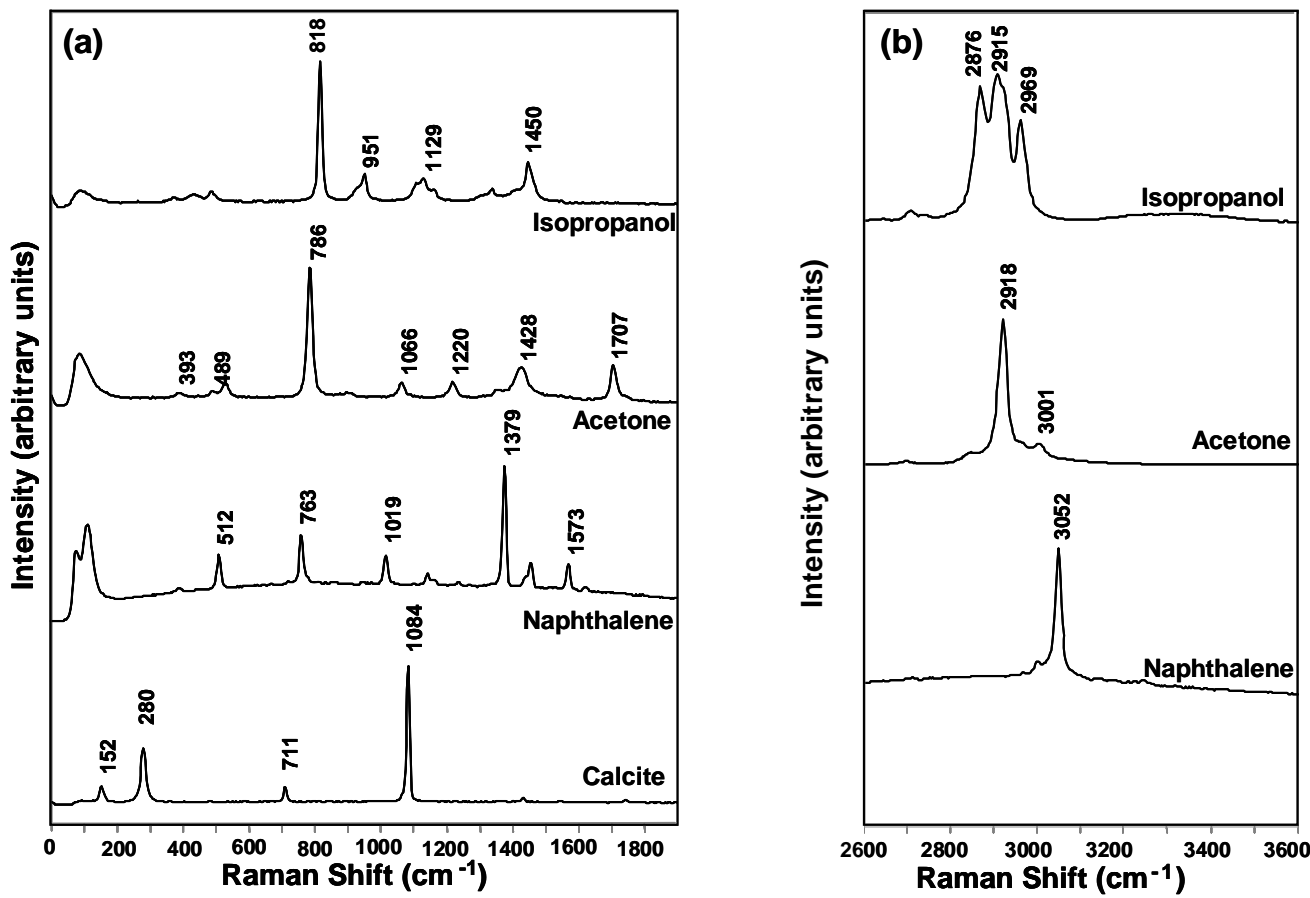

Figure 3. Raman spectra of isopropanol, acetone, naphthalene, and calcite in the (a) low frequency $\left(0-1900 \mathrm{~cm}^{-1}\right)$ and in the (b) high frequency $\left(2600-3600 \mathrm{~cm}^{-1}\right.$ ) frequency shift regions (obtained using the previous remote Raman sensor system). 
The same set of samples was measured with the current compact system, as can be seen in Figure 4a. The Raman bands caused by the stretching modes of water are evident in each sample. Even with the amount of background present in the spectra, the Raman bands are easily distinguishable. The difference between the spectrum of water and ice can also be observed, with ice being slightly shifted towards the lower frequency. In addition, water revealed a strong, sharp peak around $3120 \mathrm{~cm}^{-1}$. Formation of stronger hydrogen bond in ice makes its structure less dense than water, resulting in a decrease in the frequency of the symmetric $\mathrm{O}-\mathrm{H}$ stretching mode of water molecules in ice.

The Raman spectra of melanterite $\left(\mathrm{FeSO}_{4} \cdot 7 \mathrm{H}_{2} \mathrm{O}\right)$ and epsomite $\left(\mathrm{MgSO}_{4} \cdot 7 \mathrm{H}_{2} 0\right)$, contain broad overlapping $\mathrm{O}-\mathrm{H}$ bands in the $\mathrm{O}-\mathrm{H}$ stretching region. Micro-Raman study of these minerals has been reported with the same spectral features. ${ }^{11}$ Neutron diffraction studies of $\mathrm{CoSO}_{4} \cdot n \mathrm{D}_{2} \mathrm{O}\left(\mathrm{n}=4\right.$ and 7) and $\mathrm{MgSO}_{4} \cdot 4 \mathrm{H}_{2} \mathrm{O}$ revealed seven and four different types of crystallographic distinct, asymmetric water molecules in $\mathrm{CoSO}_{4} \cdot 7 \mathrm{D}_{2} \mathrm{O}$ and $\mathrm{CoSO}_{4} \cdot 4 \mathrm{D}_{2} \mathrm{O}$, respectively. It is believed that these hydrates qualitatively reflect the structures of the $\mathrm{X}_{2} \mathrm{O}$ molecules and $\mathrm{H}$-bonding in the melanterite and epsomite hydtrates. ${ }^{12,13}$

Raman spectrum of bischofite $\left(\mathrm{MgCl}_{2} \cdot 6 \mathrm{H}_{2} \mathrm{O}\right)$, shows two distinct $\mathrm{O}-\mathrm{H}$ stretching band peaks at 3348 and $3509 \mathrm{~cm}^{-1}$ indicating the presence of at least two distinct types of water molecules. The monoclinic structure of this mineral can be considered to be composed of $\mathrm{Cl}^{-}\left(\mathrm{H}_{2} \mathrm{O}\right)_{3} \mathrm{Mg}^{2+}\left(\mathrm{H}_{2} \mathrm{O}\right)_{3} \mathrm{Cl}$ groups with crystallographic point group symmetry $2 / \mathrm{m}$, as indicated by Neutron diffraction study of $\mathrm{MgCl}_{2} \cdot 6 \mathrm{H}_{2} \mathrm{O} .{ }^{14}$ In the high frequency spectrum of gypsum $\left(\mathrm{CaSO}_{4} \cdot 2 \mathrm{H}_{2} \mathrm{O}\right)$ shown in Figure 2b, two very sharp bands at 3401 and $3490 \mathrm{~cm}^{-1}$ are also observed, indicating the presence of two distinct types of chemically bonded water molecules in the mineral. ${ }^{15}$
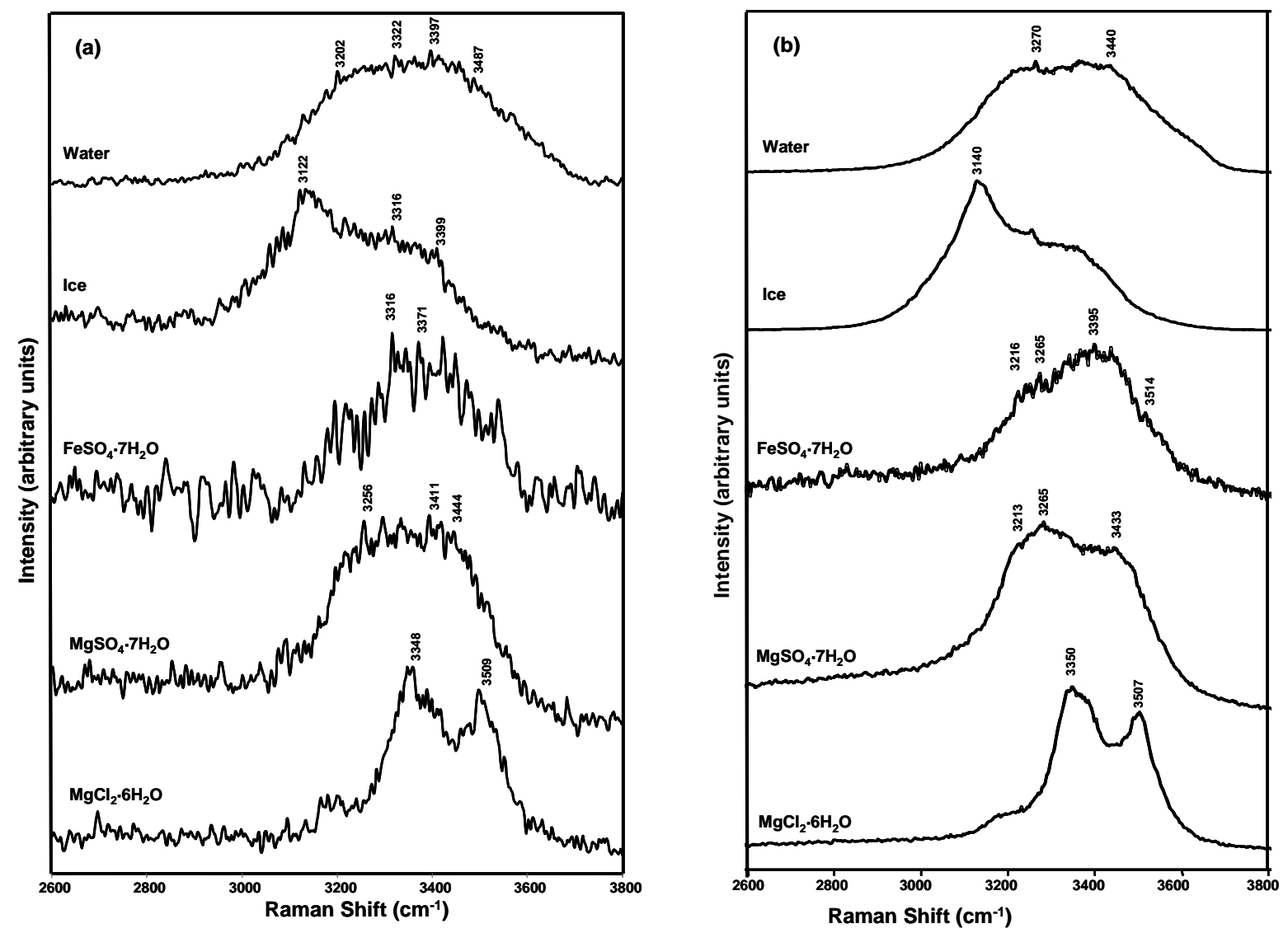

Figure 4. Raman spectra of water, ice, melanterite $\left(\mathrm{FeSO}_{4} \cdot 7 \mathrm{H}_{2} \mathrm{O}\right)$, epsomite $\left(\mathrm{MgSO}_{4} \cdot 7 \mathrm{H}_{2} \mathrm{O}\right)$, and $\left(\mathrm{MgCl}_{2} \cdot 6 \mathrm{H}_{2} \mathrm{O}\right)$ obtained using (a) improved compact remote Raman sensor system, and (b) previous remote Raman sensor system, in the high frequency region showing overlapping $\mathrm{O}-\mathrm{H}$ bands in the $3000-3600 \mathrm{~cm}^{-1}$ region. 


\section{CONCLUSION}

Modifications to the NASA Langley Research Center remote Raman sensor system was made to reduce its overall size and weight and to improve its portability. A smaller spectrograph and a mini ICCD camera were utilized, and the performance of the resulting compact system was tested for its performance. The Raman spectra of various rocks and minerals obtained with the compact system clearly showed the relevant fingerprint bands and unambiguously identified the observed materials. Similarly, with water, ice, and other water-bearing minerals, Raman bands caused by the stretching modes of $\mathrm{O}-\mathrm{H}$, were definitely observed. However, when compared to those obtained with the previously reported system, the Raman spectra obtained with the compact system contain more background signal. Furthermore, with the compact Raman system, daylight or room light operation was found to influence the ICCD camera. The problems mentioned are attributed to the limitations of the new camera system. The smaller camera system cannot be operated with microsecond or nano-second range gate width, and it cannot perform shot accumulation by software. Therefore, in order to improve the quality of the spectra, a few modifications are proposed. Firstly, an external programmable timing generator must be used for proper gated operation of the ICCD camera. Although the camera itself is able to operate using gate widths less than $500 \mathrm{~ns}$, it is the manufacturer-supplied application software that limits the gated performance of the camera. Secondly, the software will have to be modified to allow shot accumulation by software and not on the CCD itself. This modification should permit higher number of accumulation, therefore longer integration time.

\section{REFERENCES}

[1] S. K. Sharma, "New trends in telescopic remote Raman spectroscopic instrumentation," Spectrochim. Acta Part A, 68A(4), 1008-1022, (2007).

[2] J. Popp, M. Schmitt, "Raman spectroscopy breaking terrestrial barriers!,” Journal of Raman Spectroscopy, 35(6), 429-432, (2004).

[3] A. Wang, L. A. Haskin, A. L. Lane, T. J. Wdowiak, S. W. Squyres, R. J. Wilson, L. Howland, K. Manatt, N. Raouf, and C. D. Smith, "Development of Mars microbeam Raman Spectrometer,” J. Geophys. Res., 108(E1), 5005 (2003).

[4] S. K. Sharma, S. M. Angel, M. Ghosh, H. W. Hubble, P. G. Lucey, "Remote pulsed Raman spectroscopy system for mineral analysis on planetary surfaces to 66 Meters,” App. Spectrosc., 56, pp. 699-704, (2002).

[5] S. K. Sharma, A. K. Misra, P. G. Lucey, S. M. Angel, C. P. McKay, " Remote pulsed Raman spectroscopy system of Inorganic and organic materials to a radial distance of 100 meters,” App. Spectrosc., 60(8), 871-876, 2006.

[6] C. S. Garcia, M. N. Abedin, S. K. Sharma, A. K. Misra, S. Ismail, U. N. Singh, T. F. Refaat, H. E. Elsayed-Ali, S. P. Sandford, "Remote pulse laser Raman spectroscopy system for detecting water, ice, and hydrous minerals," Proc. SPIE, 6302, 630215/1-630215/7, 2006.

[7] C. S. Garcia, M. N. Abedin, S. K. Sharma, A. K. Misra, S. Ismail, , S. P. Sandford, H. E. Elsayed-Ali "Remote Raman sensor system for testing of rocks and minerals,” Proc. SPIE, 6538, 65381I1-65381I9, 2007.

[8] A. K. Misra, S. K. Sharma, C. H. Chio, P. G. Lucey, B. Lienert, "Pulsed remote Raman system for daytime measurements of mineral spectra,” Spectrochim. Acta Part A, 61, 2281-2287, 2005.

[9] NIST Standard Reference Database Website: http://webbook.nist.gov/chemistry.

[10] A. K. Misra, S. K. Sharma, C. H. Chio and P. G. Lucey, Detection of water and water bearing minerals from $10 \mathrm{~m}$ distance under bright condition using remote Raman system, Lunar and Planetary Sci. Conference, 37, abstract \#2155 (2006).

[11] C. H. Chio, Shiv K. Sharma, and David W. Muenow, The hydrates and deuterates of ferrous sulfate $\left(\mathrm{FeSO}_{4}\right)$ : a Raman spectroscopic study, J. Raman Spectrosc. 38(1), 87-99, 2006.

[12] W. H. Baur, On the crystal chemistry of salt hydrates. III. The determination of the crystal structure of $\mathrm{FeSO}_{4} \cdot 7 \mathrm{H}_{2} \mathrm{O}$ (melanterite). Acta Cryst., 17, 1167, 1964.

[13] T .Kellersohn, R.G. Delaplane and I. Olovsson, Disorder of a trigonally planar coordinated water molecule in cobalt sulfate heptahydrate, $\mathrm{CoSO}_{4} .7 \mathrm{D}_{2} \mathrm{O}$ (Bieberite), $Z$. Naturforsch., B 46, 1635, 1991.

[14] P. A. Agron and W. R. Busing, Magnesium dichloride hexahydrate, $\mathrm{MgCl}_{2} \cdot 6 \mathrm{H}_{2} \mathrm{O}$, by neutron diffraction, Acta Cryst., C41, 8-10, 1985.

[15] C. H. Chio, S. K. Sharma, and D. W. Muenow, Raman spectroscopic studies of gypsum between 33 and 374 K, Amer. Mineral., 89, 390, 2004. 